\title{
Bienestar Espiritual de los Estudiantes de Enfermería
}

\author{
Spiritual Well-Being of Nursing Students \\ Bem-Estar Espiritual de Alunos de Enfermagem
}

\author{
Elizabeth Fajardo-Ramos ${ }^{1}$, Angela María Henao-Castaño ${ }^{2}$ \\ ${ }_{1}^{1}$ Profesora Titular. Facultad de Ciencias de la Salud Universidad del Tolima. \\ Correo electrónico:efajardo@ut.edu.co \\ ${ }_{2}^{2}$ Profesora Asistente. Facultad de Enfermería Universidad Nacional de \\ Colombia. Correo electrónico: angmhenaocas@unal.edu.co \\ Cómo citar este artículo en edición digital: Fajardo-Ramos, E. \& Henao-Castaño, AM. (2019). Bienestar \\ Espiritual de los Estudiantes de Enfermería. Cultura de los Cuidados (Edición digital), 23 (54). \\ Recuperado de http://dx.doi.org/10.14198/cuid.2019.54.03 \\ Correspondencia: Universidad del Tolima. Ibagué (Colombia) \\ Correo electrónico de contacto: efajardo@ut.edu.co \\ Recibido: 09/12/2018; Aceptado: 11/03/2019
}

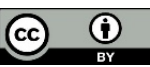

\section{ABSTRACT}

Objective: To determine the spiritual wellbeing (BE) of nursing students in a public university in the department of Tolima, Colombia.

Method: Quantitative, descriptive, exploratory, cross-sectional study. Sampling at convenience constituted by 264 nursing students of a higher education institution of the city of Ibague-Tolima, to whom the instrument SHALOM-3 was applied, to measure the spiritual well-being (dissonance and harmony) in the personal domains, transcendental, environmental and communal.

Results: The sample was constituted by women in 63.6\% (168), singles 90\% (238) and $65 \%$ (172) with ages between 16 and 21 years, of catholic religion $66.5 \%$ (175), with high Percentages in the levels of harmony between the domains, It is emphasized that the strategies that promote spiritual wellbeing in the students were the family, being happy, time to relax, music and spirituality in life.

Conclusion: These findings could be used to lay the foundations for greater support in promoting the spiritual well-being of nursing students in areas in which they presented dissonance.

Keywords: Spiritual bell-being, SHALOM, nursing, student.

\section{RESUMO}

Objetivo: Determinar o bem-estar espiritual (BE) de estudantes de enfermagem em uma universidade pública do departamento de Tolima, Colômbia.

Método: Estudo quantitativo, descritivo, exploratório, transversal. amostra de conveniência composta por 264 estudantes de enfermagem de uma instituição de ensino superior na cidade de Ibagué Tolima, ao qual foi aplicado o instrumento SHALOM-3 para medir o bem-estar espiritual (dissonância e harmonia) nos domínios pessoais, transcendental, ambiental e comunal. 
Resultados: A amostra foi constituída por mulheres em $63,6 \%$ (168), solteiros $90 \%$ (238) e $65 \%$ (172) com idades entre 16 e 21 anos, de religião católica 66,5\% (175), com alta Porcentagens nos níveis de harmonia entre os domínios, Ressalta-se que as estratégias que promovem o bem-estar espiritual nos alunos foram a família, ser feliz, tempo para relaxar, música e espiritualidade na vida.

Conclusão: Estes achados poderiam ser usados para estabelecer as bases para um maior apoio na promoção do bem-estar espiritual dos estudantes de enfermagem em áreas em que eles apresentavam dissonância.

Palavras chave: Bem-estar espiritual, SHALOM, enfermagem, estudante.

\section{RESUMEN}

Objetivo: Determinar el bienestar espiritual (BE) de estudiantes de enfermería en una universidad pública del departamento del Tolima, Colombia.

Método: Estudio cuantitativo, descriptivo, exploratorio, transversal. Muestreo a conveniencia constituido por 264 estudiantes de enfermería de una institución de educación superior de la ciudad de Ibagué- Tolima, a los cuales se les aplicó el instrumento SHALOM-3, para medir el bienestar espiritual (disonancia y armonía) en los dominios personal, trascendental, ambiental y comunal.

Resultados: La muestra fue constituida por mujeres en un 63.6\% (168), solteros 90\% (238) y el 65\% (172) con edades entre 16 y 21 años, de religión católica $66,5 \%$ (175), con altos porcentajes en los niveles de armonía entre los dominios, se resalta que las estrategias que fomentan el bienestar espiritual en los estudiantes fueron la familia, estar feliz, el tiempo para relajarse, la música y la espiritualidad en la vida.
Conclusión: Estos hallazgos podrían utilizarse para sentar las bases de un mayor apoyo en el fomento del bienestar espiritual de los estudiantes de enfermería en aspectos en los cuales presentaron disonancia.

Palabras clave: Bienestar espiritual, SHALOM, enfermería, estudiante.

\section{INTRODUCCIÓN}

La espiritualidad es una forma de encontrar esperanza significado y propósito en el mundo. Es especialmente importante cuando las personas se sienten vulnerables, cuando enfrentan enfermedades y crisis, ya sea como paciente o como enfermera (Rogers \& Wattis, 2015). El Bienestar Espiritual (BE) se describe como la naturaleza interconectiva, que incluye la armonía del ser humano originada en la congruencia entre el significado expresado y el vivido, los propósitos y los valores en la vida a nivel personal. Para el presente estudio se acoge la propuesta teórica de Fisher quien desarrollo el Instrumento SHALOM el cual ha sido sometido a rigurosas pruebas estadísticas en varios idiomas. SHALOM se ha utilizado con estudiantes de escuelas y universidades, maestros, enfermeras, médicos, asistentes a iglesias, en entornos industriales y de negocios, con mujeres maltratadas, jóvenes con problemas y alcohólicos (Fisher, 2010).

Incluye los conceptos de Armonía Espiritual y Disonancia espiritual. El concepto denominado Armonía Espiritual se refiere al estado de bienestar espiritual que se encuentra en congruencia con la percepción acerca de los valores $\mathrm{y}$ propósitos de vida; por el contrario la Disonancia Espiritual se define como la diferencia marcada entre las experiencias ideales y aquellas vividas en los dominios 
del BE (J. Fisher, 2010).

El BE espiritual para Fisher es "un estado dinámico del ser, caracterizado por la medida en que las personas viven en armonía o disonancia en los dominios: personal, comunal, ambiental y trascendental". El dominio personal se define como la percepción de bienestar de cada persona consigo misma y con respecto al significado, al propósito y a los valores en la vida. El dominio comunal es la forma en que se muestran la calidad y la profundidad de las relaciones interpersonales, entre el yo y los otros, en relación a la moral, a la cultura y a la religión; expresados en el amor, en el perdón, en la confianza, en la esperanza y en la fe en la humanidad. El dominio ambiental va más allá del cuidado de la vida y el ambiente, en un sentido de admiración y asombro por la naturaleza, incluye, la noción de unidad física y biológica con el medio ambiente. El dominio trascendental es la relación del yo con algo o alguien más además del nivel humano, o sea, una fuerza cósmica superior, la realidad transcendente o Dios.

Es importante en la práctica de enfermería brindar bienestar a las personas a través de las actividades de cuidado y en respuesta se recibe una recompensa espiritual que refuerza los valores y ofrece la oportunidad de proyectarse personal y profesionalmente, al dar cuidado humano por medio de la reciprocidad se generan las relaciones interpersonales terapéuticas. En este sentido, se justifica el valor de la dimensión transpersonal de beneficiar al otro $y$ enriquecerse en el proceso de dar cuidado (Gómez \& Fisher, 2003; J. W. Fisher, 2008; John W. Fisher, 1999). Es así como la idea expuesta sobre el BE es el resultado del compromiso de la disciplina de enfermería con el cuidado de la persona desde una concepción humanista (J. Fisher \& Brumley, 2008).
Otro aspecto a destacar es la novedad de explorar un tema de interés para la disciplina de enfermería dado que medir el Bienestar Espiritual permite crear conciencia del BE en las personas sanas. Se realizó la presente investigación con la finalidad de determinar el BE (disonancia o armonía) de los estudiantes de enfermería, a través del instrumento SHALOM-3 considerando la propuesta teórica de John Fisher.

\section{METODOLOGÍA}

Estudio con enfoque cuantitativo, de tipo descriptivo, exploratorio, transversal. Aprobado por los comités de ética de las universidades involucradas. Para dar cumplimiento a los principios éticos de la investigación, los participantes fueron entrevistados de manera personal por el grupo de investigadoras previo cumplimiento del protocolo de investigación y consentimiento informado.

La población fue compuesta por los estudiantes del programa de enfermería de una institución de educación superior que correspondían a 280 personas del programa de enfermería de todos los cursos es decir 10 semestres de la Universidad del Tolima, en el periodo de Julio a Septiembre del 2017. El muestreo fue a conveniencia con la participación de 264 personas que cumplieron los siguientes criterios de inclusión: ser estudiantes activos del programa de enfermería. Se excluyeron aquellos estudiantes de enfermería que se encontraban con algún tipo de ausencia como incapacidad médica.

La recolección de la información se llevó a cabo con el Instrumento SHALOM-3 y el formato de datos sociodemográficos. El cuestionario fue diligenciado una vez en cada uno de los salones en los cuales los estudiantes se encontraban. El Instrumento 
de evaluación del Bienestar espiritual: Fue utilizada la versión abreviada del instrumento original llamado SHALOM-(J. Fisher, 2010), que incluye tres componentes: Evaluación de la Salud espiritual $(A)$, de la orientación de Vida (B) y de la percepción del enfermero sobre la ayuda dada a los pacientes (C); son cuestionados a través de 20 ítems correspondientes y agrupados en los cuatro dominios (personal, comunal, ambiental y transcendental), por medio de tres preguntas a ser respondidas con escala tipo Likert de 1 (muy poco) hasta 5 (muchísimo).

El componente $A$ muestra los "ideales" de salud espiritual para las personas, por medio de la pregunta: ¿Qué tan importante es cada uno de los siguientes elementos para conseguir un estado ideal de salud espiritual? En el componente $B$ es preguntado: ¿Cómo usted siente que los siguientes temas reflejan su experiencia personal la mayor parte del tiempo?

Finalmente es cuestionado el nivel de contribución de diferentes estrategias que fomentan el BE en la persona, por ejemplo: familia, oración, caminatas, tiempo para relajamiento, ayudar a los otros, etc. también usando una escala tipo Likert de 5 puntos.

Esta versión abreviada del instrumento denominada SHALOM-3, cuenta con propiedades psicométricas de validez y confiabilidad en el idioma inglés, resultado del trabajo sistemático y riguroso de confiabilidad (Alfa de Cronbach) y validez (constructo, discriminante, la independencia concurrente, predictiva y factorial de la personalidad) aplicados a cuatro estudios que soportan el modelo Fisher (Gomez \& Fisher, 2003). Es así que con relación al Alpha de Cronbach reportado en las cuatro dimensiones: personal, comunal, ambiental $\mathrm{y}$ transcendental, es de, $0.81,0.82,0.86$ e 0.95 respectivamente. El instrumento presentó validez facial, de contenido y constructo en el idioma español, lo que muestra su utilidad en el contexto hispano-hablante colombiano para determinar el Bienestar Espiritual de las personas (Henao, Angela; Molano, Maria Luisa; Fuentes, Alejandra; Gómez, Olga; Diaz, 2016).

El análisis estadístico, para las variables cuantitativas se calculó medidas de tendencia central (promedio) y de variabilidad (desviación estándar), para las variables cualitativas se calcularon proporciones o porcentajes, la mayoría de los resultados se presentaron en proporciones.

Bienestar Espiritual - BE. Con base en las respuestas de los 5 ítems para cada una de las 3 categorías (A-ideal; B-experiencia vivida "sentimiento"; C-ayuda a otros) de cada uno de los dominios (PER: personal; COM: comunal; AMB: ambiental; TRA: transcendental) y con valores reportados en una escala de 1-5, se calcularon los porcentajes y promedios de respuesta tanto en general como por semestre.

Para evaluar el BE, se calculó el porcentaje acumulado en las escalas "Alto" y "muy Alto" para las 3 categorías de cada dominio, estos porcentajes se calcularon sumando los porcentajes de las dos escalas "Alto" y "muy Alto" y promediando para las 3 categorías.

Igualmente se estimó el alfa de Cronbach para evaluar la consistencia interna de los datos en cada dominio; cuanto más se acerque a 1, mejor es la fiabilidad, considerando una buena fiabilidad a partir de 0.80 . Este coeficiente se calculó para cada categoría dentro de los cuatro dominios, en general y de manera detallada por semestre. Los valores promedios de las respuestas de los estudiantes en las 3 categorías para cada uno de los 4 dominios de BE, se calcularon dividiendo la sumatoria de los ítems de cada dominio sobre el número de ítems del dominio correspondiente, según lo indica 
Fisher, este es el cálculo recomendado cuando existen valores missing o datos perdidos, sin embargo en este estudio la tasa de datos perdidos fue muy baja correspondiente a un $11 \%$ en determinados ítems.

Teniendo en cuenta lo propuesto por Fisher donde explica la disonancia espiritual como una diferencia en promedio mayor de 1,0 (más de 1 desviación estándar) entre los "ideales" y "la experiencia vivida" en cualquier dominio de BE medido a través del instrumento SHALOM, se calcularon las diferencias promedio y posteriormente el porcentaje de Disonancia Espiritual (mayor de 1) en las 3 categorías para cada uno de los 4 dominios de $\mathrm{BE}$, de forma general y por semestre, e igualmente se estratifico por género y grupos de edad.

Las comparaciones por género y grupos de edad, se realizaron con la prueba de Chi cuadrado o prueba de Fisher según el caso. En las comparaciones se consideraron diferencias significativas para un valor $\mathrm{p}<0.05$. Finalmente se describieron las Estrategias que fomentan el bienestar espiritual en los estudiantes.

Los datos fueron almacenados en una base de datos de Excel 2007 y posteriormente analizados estadísticamente en el programa Stata versión 11.2® (College Station, Texas, USA).

\section{RESULTADOS}

Se incluyeron 264 estudiantes de la Institución Universidad del Tolima, de los cuales el 63.6\% (168) correspondieron al género femenino, el 90\% (238) son solteros y el 65\% (172) estaban entre los 16 y 21 años de edad. La mayoría de los estudiantes pertenecen a la religión católica 66,5\% (175) y cristiana $38(14,45)$.

Con base en las respuestas de los 5 ítems para cada una de los 4 (personal, comunal, ambiental y trascendental) para Bienestar Espiritual - BE, los resultados para el dominio personal, en cada uno de los 10 semestres. Se observaron altos porcentajes en las escalas "Alto" y "Muy Alto" para las 3 categorías del dominio personal, en todos los 10 semestres, cuyo valor promedio fue del $82 \%$, lo que refleja un buen bienestar en este dominio. Los valores del alfa de Cronbach del dominio Personal en general para las 3 categorías fueron $(0.81,0.81,0.83)$.

En cuanto al dominio comunal, el porcentaje promedio fue del $70 \%$ en las dos escalas "Alto" y "Muy Alto", con valores alfa de Cronbach para las 3 categorías de $(0.78,0.71,0.75)$.

El dominio ambiental presentó los altos porcentajes en las escalas "Alto" y "Muy Alto" fue del 69\% debido a que un $22 \%$ de las respuestas variaron en promedio en la escala "Moderado", los valores alfa de Cronbach de las 3 categorías fueron (0.84, $0.84,0.88)$.

Por último en el dominio trascendental, el porcentaje promedio en las escalas "Alto" y "muy Alto" fue del 62\%, donde se observó también un $24 \%$ de respuestas en la escala "Moderado", cuyos valores alfa de Cronbach de las 3 categorías fueron (0.93, 0.92, 0.93) respectivamente, siendo los más altos. Estos resultados evidencian una alta consistencia interna, sobre todo en los dominios trascendental y ambiental. La disonancia espiritual en promedio fue del $27,5 \%$ siendo un poco mayor en el dominio trascendental $(33,5 \%)$ por el contrario el dominio comunal tuvo el menor porcentaje de disonancia $(21,2 \%)$, los porcentajes de disonancia en los 4 dominios discriminando por categorías, observándose mayor disonancia entre las categorías ideal y ayuda a otros, tanto en el dominio Ambiental como trascendental. Ver figura 1: 


\section{FIGURA 1: Porcentaje de Disonancia General}

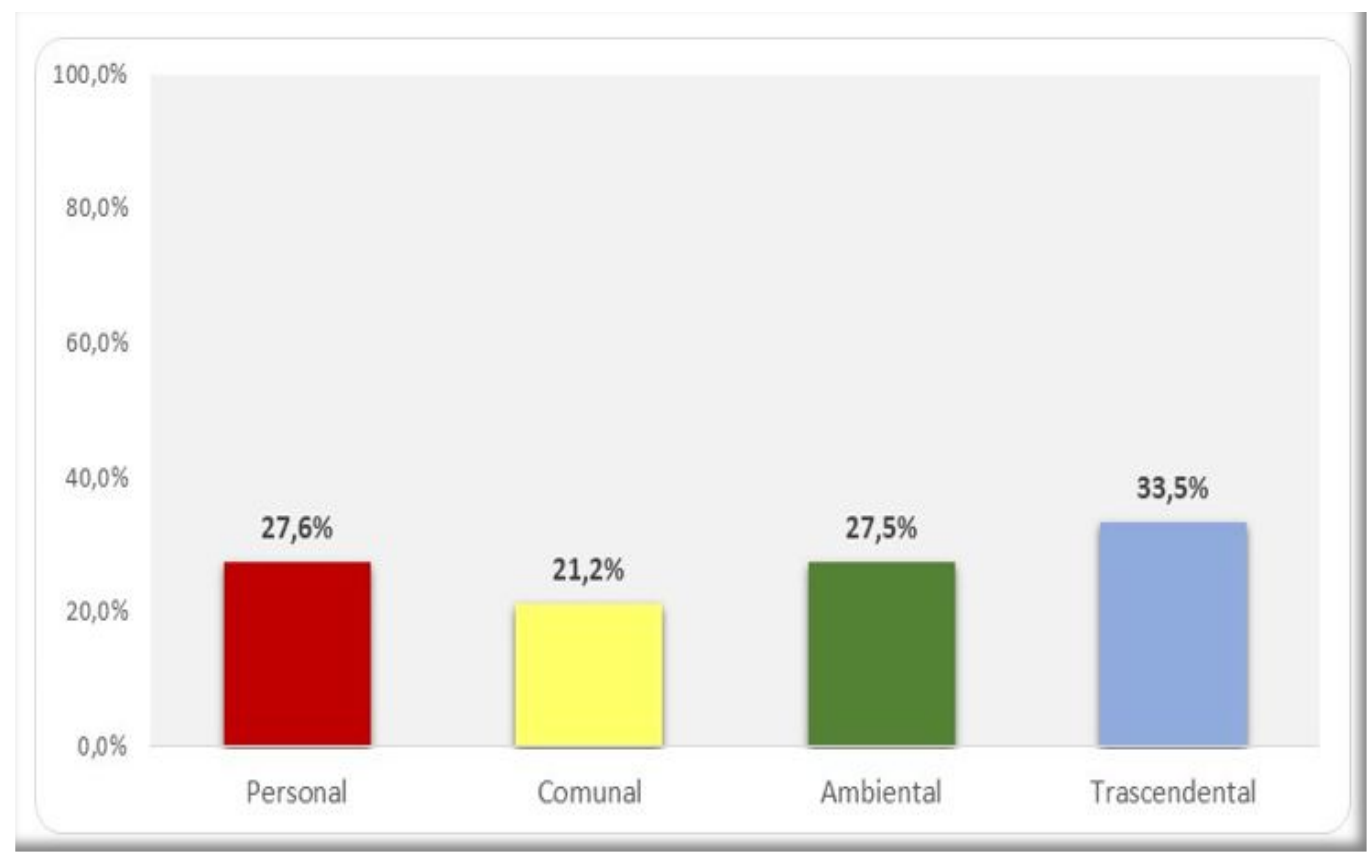

FUENTE: Resultados de Investigación. Fajardo E. Henao AM

En el dominio Personal con un porcentaje de disonancia del 27,6\% (66/239), del total de disonantes, la mayoría $(57,6 \%)$ fueron del género femenino. El $65 \%$ de los casos disonantes tenían una edad entre 16 a 21 años, seguido del grupo de 22 a 25 años con un $24 \%$. No se observaron diferencias significativas entre grupos ( $\mathrm{p}=0.96)$.

En el dominio Comunal la disonancia fue del 21,2\% (51/241), de los disonantes el 53\% fueron mujeres y la mayoría (68,6\%) también tenían una edad entre 16 y 21 años, y no hubo diferencias significativas entre grupos $(\mathrm{p}=0.69)$. En cuanto al dominio Ambiental el $27,5 \%$ tenían disonancia (65/236), de estos 41 fueron del género femenino (63\%), la mayoría tenía una edad entre 16 y 21 años $(65 \%)$. No se observaron diferencias significativas entre grupos $(\mathrm{p}=0.78)$.
Por último, en el dominio trascendental la disonancia fue del 33,5\% (80/239), de los cuales el $57,5 \%$ fueron mujeres, y el $64 \%$ también estaban entre 16 y 21 años, seguido de un $27,5 \%$ de 22 a 25 años, no hubo diferencias significativas ( $\mathrm{p}=0.84$ ).

Estrategias para fomentar el Bienestar Espiritual, en la tabla 1, se presentan las estrategias que fomentan el bienestar espiritual en los estudiantes, para ellos la familia, el estar feliz, tiempo para relajarse, la música y ayudar a los demás, fueron las estrategias más usuales en las escalas de mayor frecuencia "Alto" y "Muy Alto".

Por el contrario, las estrategias relacionadas con el pastor (sacerdote, líder religioso) y literatura sagrada, fueron las menos usuales, con mayor porcentaje en "Muy poco" y "Poco". 
TABLA 1: Estrategias que fomentan el bienestar espiritual de los estudiantes (Orden descendente por la escala "Muy alto")

\begin{tabular}{lrrrrr}
\hline Estrategia & Muy poco & Poco & Moderado & Alto & Muy Alto \\
\hline Familia & 2,39 & 2,39 & 14,74 & 26,69 & 53,78 \\
\hline Estar feliz & 1,59 & 3,19 & 15,14 & 26,29 & 53,78 \\
\hline Tiempo para relajarse & 1,59 & 5,18 & 17,53 & 28,69 & 47,01 \\
\hline Música & 2,39 & 7,17 & 20,32 & 27,89 & 42,23 \\
\hline Ayudar a los demás & 2,79 & 4,38 & 18,33 & 40,24 & 34,26 \\
\hline La espiritualidad en su vida & 3,59 & 7,57 & 22,31 & 32,27 & 34,26 \\
\hline Naturaleza & 4,78 & 6,77 & 21,91 & 33,86 & 32,67 \\
\hline Mejoramiento personal & 1,2 & 3,19 & 21,12 & 45,82 & 28,69 \\
\hline Oración & 10,36 & 17,93 & 20,32 & 23,9 & 27,49 \\
\hline Deportes & 8,37 & 13,55 & 32,67 & 18,33 & 27,09 \\
\hline Caminatas & 5,98 & 8,76 & 29,48 & 33,07 & 22,71 \\
\hline Meditación & 10,36 & 19,12 & 25,1 & 23,9 & 21,51 \\
\hline Amigos & 3,98 & 4,78 & 30,68 & 39,44 & 21,12 \\
\hline La religión en su vida & 17,13 & 15,54 & 27,49 & 21,51 & 18,33 \\
\hline La iglesia, actividad religiosa & 19,52 & 17,53 & 27,89 & 19,92 & 15,14 \\
\hline Consejería & 10,76 & 18,33 & 33,47 & 23,51 & 13,94 \\
\hline Literatura sagrada & 25,5 & 23,9 & 27,49 & 11,95 & 11,16 \\
\hline Pastor, sacerdote, líder religioso & 37,85 & 26,69 & 20,32 & 5,98 & 9,16 \\
\hline
\end{tabular}

FUENTE: Resultados de Investigación. Fajardo E. Henao

\section{Discusión}

El bienestar espiritual se refleja en la calidad de las relaciones que las personas tienen consigo mismas, con los demás, con el entorno y / o con Dios (J. Fisher, 2010) los hallazgos de este estudio sugieren un panorama alentador del bienestar espiritual que presentan los estudiantes de enfermería, queda por explorar si con el paso de los semestres en el plan de estudio se mantienen estos altos porcentajes en cada uno de los participantes.

Estos resultados pueden explicarse por el hecho de que la mayoría eran religiosos, igualmente contaban con diferentes estrategias individuales que fomentan el bienestar espiritual tales como la familia, estar feliz, la música, tiempo para relajarse y la espiritualidad en su vida (Ross et al., 2014).

Abordar este tema en enfermeros en formación es relevante dado que existe una importante relación entre el bienestar espiritual que puede llegar a alcanzar el enfermero en el ámbito laboral y la actitud que cada uno tiene frente a la atención espiritual de las personas de cuidado y compromiso profesional (Chiang, Lee, Chu, Han \& Hsiao, 2016).

La evaluación del Bienestar espiritual en los 
estudiantes de enfermería es un tema que se ha venido estudiando, especialmente la inclusión de esta temática en los planes de estudio de los programas de enfermería dada la importancia para el enfermero en formación y su incorporación del concepto en la práctica así como el uso de escalas de evaluación espiritual (Helming, 2009) (Wu, Tseng \& Liao, 2016).

Esta preocupación en relaciona a la espiritualidad se presenta dado los hallazgos de estudios en los cuales sugieren que las enfermeras brindan cuidados espirituales con poca frecuencia, esta evidencia hace un llamado a mejorar la educación y el apoyo de la atención espiritual para las enfermeras (Taylor, Mamier, Ricci-Allegra \& Foith, 2017).

En esta misma línea los órganos gubernamentales reconocen el trabajo de la práctica avanzada de enfermería en consonancia con la atención espiritual de los pacientes lo que llevo el aumento del entrenamiento de los enfermeros en temas de bienestar espiritual lo que llevo una reducción de incomodidad con la provisión de cuidados espirituales (DeKoninck, Hawkins, Fyke, Neal \& Currier, 2016; Preposi, Alshammari, Aied \& Colet, 2017).

\section{CONCLUSIONES}

Resaltar la importancia del bienestar espiritual en el enfermero en formación y como profesional ha permitido identificar aspectos positivos tanto de manera individual como con la persona objeto de cuidado, sin embargo quedan vacíos que se deben mitigar en los acercamiento de experiencias de aprendizaje personales para los estudiantes de enfermería proporcionándoles las herramientas necesarias para la implementación de estrategias que fomenten el bienestar espiritual al interior de la universidad. Los hallazgos de este estudio de investigación proporcionan pautas para una de esas experiencias de aprendizaje y evidencia de su efectividad.

\section{REFERENCIAS}

- Chiang, Y. C., Lee, H. C., Chu, T. L., Han, C. Y. \& Hsiao, Y. C. (2016). The impact of nurses' spiritual health on their attitudes toward spiritual care, Professional commitment, and caring. Nursing Outlook, 64(3), 215-224.

- DeKoninck, B., Hawkins, L. A., Fyke, J. P., Neal, T. \& Currier, K. (2016). Spiritual Care Practices of Advanced Practice Nurses: A Multinational Study. Journal for Nurse Practitioners, 12(8), 536-544. Recuperado de https://doi.org/10.1016/j.nurpra.2016.05.023.

- Fisher, J. (2010). Development and Application of a Spiritual Well-Being Questionnaire Called SHALOM. Religions, 1(1), https://doi.org/10.3390/rel1010105.

- Fisher, J. \& Brumley, D. (2008). Nurses' and carers' spiritual wellbeing in the workplace. Australian Journal of Advanced Nursing 25(4).

- Fisher, J. W. (1999). Helps to fostering students' spiritual health [1]. International Journal of Children's Spirituality, $4(1), \quad 29-49$. https://doi.org/10.1080/1364436990040104.

- Fisher, J. W. (2008). Impacting teachers' and students' spiritual well-being. Journal of Beliefs \& Values, 29(3), 253-261. Recuperado de https://doi.org/10.1080/13617670802465789.

- Gómez, R. \& Fisher, J. W. (2003). Domains of spiritual well-being and development and validation of the Spiritual Well-Being Questionnaire. Personality and Individual Differences, 35(8), 1975-1991. Recuperado de https://doi.org/10.1016/S0191-8869(03)00045-X.

- Helming, M. (2009). Integrating Spirituality Into Nurse Practitioner Practice: The Importance of Finding the time. Journal for Nurse Practitioners, 5(8), 598-605.

- Henao, Angela; Molano, Maria Luisa; Fuentes, Alejandra; Gómez, Olga; Diaz, L. P. (2016). Shalom una herramienta valida para medir el cuidado espiritual. In UN (Ed.), Experiencias de formación, investigación y práctica en el cuidado de enfermería. (1st ed., p. 352).

- Preposi, J., Alshammari, F., Aied, K. \& Colet, P. C. (2017). Nurse Education Today Spirituality and 


\section{Cultura de los Cuidados}

spiritual care perspectives among baccalaureate nursing students in Saudi Arabia: A cross-sectional study. Nurse Education Today, 49, 156-162. Rogers, M. \& Wattis, J. (2015). Spirituality in nursing practice. Nursing Standard, 29(39), 51-57.

- Ross, L., Van Leeuwen, R., Baldacchino, D., Giske, T., McSherry, W., Narayanasamy, A., Schep-Akkerman, A. (2014). Student nurses perceptions of spirituality and competence in delivering spiritual care: A European pilot study. Nurse Education Today. 34(5):697-702.

- Taylor, E. J., Mamier, I., Ricci-Allegra, P. \& Foith, J.
(2017). Self-reported frequency of nurse-provided spiritual care. Applied Nursing Research, 35, 30-35. Recuperado de https://doi.org/10.1016/j.apnr.2017.02.019.

- Wu, L., Tseng, H. \& Liao, Y. (2016). Nurse Education Today Nurse education and willingness to provide spiritual care. Nurse Education Today, 38, 36-41. Recuperado de https://doi.org/10.1016/j.nedt.2016.01.001. 